J. Clin. Chem. Clin. Biochem.

Vol. 26, 1988, pp. 569-572

(C) 1988 Walter de Gruyter \& Co.

Berlin - New York

\title{
Ein neuer immunoluminometrischer Festphasen-Sandwich-Assay zum quantitativen Nachweis von Antikörpern gegen das Hepatitis B-Oberflächen $\left(\mathrm{HB}_{\mathrm{s}}\right)$-Antigen
}

\author{
Von U. Missler und W. G. Wood \\ Klinische Laboratorien, Klinik für Innere Medizin (Direktor: Prof. P. C. Scriba), Medizinische Universität zu \\ Lübeck
}

(Eingegangen am 15. Dezember 1987/26. Mai 1988)

Zusammenfassung: Die Entwicklung eines immunoluminometrischen Festphasen-Assays zur quantitativen Bestimmung von Antikörpern gegen das Hepatitis B-Oberflächen-Antigen wird beschrieben.

Wir entwickelten einen Immunoassay, der sich Polystyrol-Kugeln von $6.4 \mathrm{~mm}$ Durchmesser als Festphase bedient. Zur Beschichtung der Kugeln benutzten wir hochgereinigtes, aus dem Plasma von chronischen $\mathrm{HB}_{\mathrm{s}}-$ Antigen-Trägern gewonnenes $\mathrm{HB}_{\mathrm{s}}$-Antigen. Nach Inkubation mit Serum für $1,5 \mathrm{~h}$ wurde wiederum $\mathrm{HB}_{\mathrm{s}}$ Antigen, das mit dem Luminogen 7-(4-Aminobutyl-N-ethyl)-naphthalin-1,2-dicarbonsäure-hydrazid-hemisuccinamid (ABEN-H) markiert war, zum Nachweis gebundener Antikörper verwendet. Diese zweite Inkubation dauerte eine Stunde. Notwendige Standard- bzw. Probenverdünnungen stellten wir in Seren her, die als negativ für Anti-HB $\mathrm{B}_{\mathrm{s}}$ bestimmt worden waren.

Die Nachweisgrenze des Verfahrens liegt bei etwa 1,3 U/1, die obere Grenze des Meßbereiches bei $1000 \mathrm{U} / 1$ mit einem mittleren Variationskoeffizienten (VK) von 5\%. Der Dynamik-Bereich (Impulsverhältnis zwischen negativen Seren und höchstem Standard) liegt bei 182:1 (4200:23 Relative Light Units). Das Verfahren

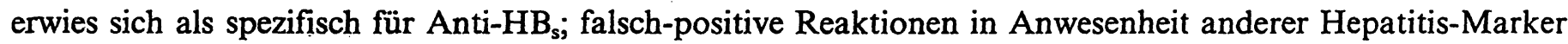
traten in den geprüften Seren nicht auf.

$A$ new immunoluminometric solid phase sandwich assay for antibodies to hepatitis $B$ surface antigen

Summary: A solid phase quantitative immunoluminometric assay for antibodies to hepatitis $B$ surface antigen (Anti-HB $\mathrm{H}_{\mathrm{s}} \mathrm{Ag}$ ) is described.

This solid phase assay uses polystyrene balls of $6.4 \mathrm{~mm}$ diameter, coated with pure hepatitis $B$ surface antigen $\left(\mathrm{HB}_{\mathrm{s}} \mathrm{Ag}\right.$ ) obtained from the plasma of chronic $\mathrm{HB}_{\mathrm{s}} \mathrm{Ag}$ carriers. After incubation with patient serum $(90 \mathrm{~min})$, $\mathrm{HB}_{\mathrm{s}} \mathrm{Ag}$ labelled with 7-(4-aminobutyl-N-ethyl)naphthalene 1,2-dicarboxylic acid hydrazide hemisuccinamide (ABEN-H) wàs used as the signal carrier.

Dilutions of samples and standards were made with sera previously shown to be negative for anti $\mathrm{HB}_{\mathrm{s}} \mathrm{Ag}$. The lower detection limit was about $1.3 \mathrm{U} / \mathrm{l}$ the upper limit for undiluted serum about $1000 \mathrm{U} / \mathrm{l}$. The mean coefficient of variation was $5 \%$.

The dynamic range (expressed as the signal ratio of the highest standard: zero standard) was 182:1 and covered the range $23-4200$ relative light units.

The assay was tested for specificity and gave no false-positive reactions in the presence of other hepatitis B antigens and antibodies. 


\section{Einführung}

Antikörper gegen das Hepatitis B-Oberflächen (Surface)-Antigen ( $\mathrm{HB}_{\mathrm{s}}$-Antigen) werden seit Jahren, wie schon 1973 von Ginsberg et al. $(1,2)$ beschrieben, routinemäßig nachgewiesen. Über die Bedeutung der Antikörper gegen das $\mathrm{HB}_{\mathrm{s}}$-Antigen, insbesondere im Zusammenhang mit der Immunantwort auf die aktive Hepatitis B-Impfung, wurde bereits an anderer Stelle berichtet $(3-8)$.

Unser Ziel war es, den Verlauf der Immunantwort auf die Hepatitis B-Impfung mittels eines immunoluminometrischen Assays (ILMA) zu untersuchen.

Da die Anti- $\mathrm{HB}_{\mathrm{s}}-$ Konzentration von nicht nachweisbar bis zu mehreren Hunderttausend Einheiten pro Liter reichen und die zur Zeit zur Verfügung stehenden

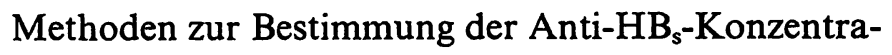
tionen maximal $200 \mathrm{U} / 1$ messen, wurde versucht, ein Verfahren mit größerem Meßbereich zu entwickeln.

\section{Material und Methoden}

Polystyrol-Kugeln: Firma Spherotech Kugeln, D-6400 Fulda.

Das $\mathrm{HB}_{\mathrm{s}}$-Antigen wurde von uns von der Universität Göttingen und den Behringwerken, AG, Marburg zur Verfügung gestellt (Typ ad, ay).

7-(4-Aminobutyl-N-ethyl)-naphthalin-1,2-dicarbonsäure-hydrazid (ABEN) wurde im Hause synthetisiert ${ }^{1}$ ), Sephadex G10-Gel stammte von Pharmacia-LKB, Uppsala, Schweden, Anti-HB $\mathrm{B}_{\mathrm{s}}$-negative Seren stellten wir aus Fresh-Frozen-PlasmaKonserven aus dem Institut für Immunologie und Transfusionsmedizin der Medizinischen Universität zu Lübeck durch Recalzifizieren her, nachdem sie sich als nicht reaktiv für Anti$\mathrm{HB}_{\mathrm{s}}$ erwiesen hatten.

Human-Serumalbumin: Firma Sigma, Taufkirchen, D.

Inkubationsplatten von Abbott Diagnostic Products, D-6200 Wiesbaden, die Meßröhrchen (Nr. 55.484 und 55.476) wurden von Sarstedt, Nümbrecht-D, bezogen. Das Waschgerät (Proquantum) stammte ebenfalls von Abbott Diagnostic Products.

Die Katalase (Nr. 106836) bezogen wir von Boehringer, Mannheim, die benutzten Luminometer (Typ LB 950 und LB 952) lieferte das Laboratorium Prof. Dr. Berthold, D-7547 Wildbad.

\section{Beschichten der Polystyrolkugeln}

Herstellung einer $0.05 \mathrm{~mol} / 1$ Phosphatpufferlösung pH 7.5 (150 $\mathrm{ml}$ pro 1000 Kugeln), Zugabe von $\mathrm{HB}_{\mathrm{s}}$-Antigen $\left(\mathrm{HB}_{\mathrm{s}}-\mathrm{Ag}\right.$ von der Universität Göttingen Subtypen ad und ay im Verhältnis $5: 1,80 \mu \mathrm{g}$ pro $1000 \mathrm{Kugeln}$ ); möglichst simultanes Einbringen der zuvor abgewogenen Menge unbehandelter Polystyrol-Kugeln (141 g pro 1000 Kugeln); Inkubation im Wasserbad für $24 \mathrm{~h}$ bei $37^{\circ} \mathrm{C}$; Nachbeschichten mit $3 \mathrm{~g} / \mathrm{l}$ Ovalbumin (No. A $5378 \mathrm{Fa}$. Sigma), Konservierung mit 0,015 mol/1 $\mathrm{NaN}_{3}$, Lagerung bei $4^{\circ} \mathrm{C}$ im Kühlschrank. 1) Interessierte können die Synthese unter der Korrespondenz-
adresse erfragen.
Herstellen des Tracers

$480 \mu \mathrm{g} \mathrm{HB}$ - $-\mathrm{Ag}$ von der Universität Göttingen Subtypen ad und ay $5: 1$; Aktivierung des 7-(4-Aminobutyl-N-ethyl)-naphthalin1,2-dicarbonsäure-hydrazid wie bereits von Wood et al. beschrieben (10) mittels Bernsteinsäureanhydrid, N-Hydroxysuccinimid und Dicyclohexylcarbodiimid in Dimethylformamid, Konzentration der resultierenden Lösung: $50 \mathrm{mmol} / \mathrm{l}$; Alkalisierung der $\mathrm{HB}_{3}-\mathrm{Ag}$ Lösung mittels $\mathrm{K}_{2} \mathrm{HPO}_{4}(0.5 \mathrm{~mol} / \mathrm{l})$ auf einen $\mathrm{pH}$ von 8.9; Zugabe von $25 \mu 1$ 7-(4-Aminobutyl-N-ethyl)naphthalin-1,2-dicarbonsäurehydrazid-Lösung, Inkubation für eine Stunde bei Raumtemperatur, anschließend Zentrifugation bei $15000 \mathrm{~min}^{-1}$ für 1 min zur Entfernung entstandenen Niederschlags; chromatographische Trennung des markierten $\mathrm{HB}_{\mathbf{s}}=$ $\mathrm{Ag}$ von ungebundenem 7-(4-Aminobutyl-N-ethyl)-naphthalin1,2-dicarbonsäure-hydrazid über eine Sephadex G10 Säule $(8 \times 300 \mathrm{~mm}) \mathrm{mit}$ Tris- $\mathrm{HCl} 0.025 \mathrm{~mol} / 1 \mathrm{pH} 7.5$ und $0.15 \mathrm{~mol} / 1$ $\mathrm{NaN}_{3}$ als Eluationspuffer; fraktioniertes Sammeln des Eluates mit einem LKB 2112 Radirac (je 15 Tropfen). Anschließend Verdünnung der Fraktionen eins bis zwanzig $1: 1000$ in phosphatgepuffertem Natriumchlorid mit $0.025 \mathrm{ml} / 1$ Tween 20, Messung von jeweils $20 \mu$ dieser Verdünnung im Luminometer; Poolung der Fraktionen mit der höchsten Impulsrate.

Der so hergestellte Tracer wurde $1: 30$ in phosphatgepuffertem $\mathrm{NaCl}-T$ ween $20+60 \mathrm{~g} / \mathrm{l}$ Human-Serumalbumin verdünnt, $\mathrm{zu}$ je $125 \mu \mathrm{l}$ portioniert und anschließend lyophilisiert. Zur Herstellung der Tracer-Gebrauchslösung wurde das Lyophilisat mit $12,5 \mathrm{ml}$ phosphatgepuffertem $\mathrm{NaCl}$-Tween $20+60 \mathrm{~g} / \mathrm{l}$ HumanSerumalbumin rekonstituiert, entsprechend einer Endverdünnung von $1: 3000$.

\section{Vorbereitung der Proben}

Proben unverdünnt mit $200 \mu \mathrm{l}$ bzw. in Verdünnungen von $1: 10$, $1: 100$ etc. in Anti-HB $B_{s}$ negativem Serum einsetzen.

Plasmen wie folgt recalzifizieren: 9 Teile Plasma +1 Teil $\mathrm{CaCl}_{2}$ $0.25 \mathrm{~mol} / \mathrm{l}$, zwei Stunden Inkubation im Wasserbad bei $37^{\circ} \mathrm{C}$, Zentrifugation bzw. bei größeren Volumina bei $-80^{\circ} \mathrm{C}$ tieffrieren, wieder auftauen und das retrahierte Fibringerinnsel entfernen (aus Beschreibung des AUSAB EIA, Abbott).

\section{Gewinnung der Standards}

Gewinnung von Plasma mittels Plasmapherese aus dem Blut eines Impflings zwei Jahre nach abgeschlossener Immunisierung mit HB-Vax (MSD-Behring), das wie oben beschrieben recalzifiziert, zu $5 \mathrm{ml}$ portioniert tiefgefroren, anschließend lyophilisiert und bei $-80^{\circ} \mathrm{C}$ gelagert wurde.

Resolubilisierung mit jeweils $5 \mathrm{ml} \mathrm{H}_{2} \mathrm{O}$, Kalibrierung am Anti$\mathrm{HB}_{\mathrm{s}}$-Standard des Paul-Ehrlich-Instituts (Herstellen nach Vorschrift des Paul Ehrlich-Instituts) durch achtfach-Bestimmung in verschiedenen Verdünnungen, so bestimmte Anti-HB s $_{\mathbf{s}} \mathrm{Kon}$ zentration des eigenen Standards: $3000 \mathrm{U} / 1$ (mehrfach bestätigt in Wiederholungsmessungen).

Erstellen einer Standardreihe mit folgenden Anti-HB $\mathrm{H}_{\mathrm{s}}-\mathrm{K}_{\mathrm{K}}$ onentrationen:
1: $1,3 \mathrm{U} / 1 \quad 5: 111 \mathrm{U} / 1$
2: $4,1 \mathrm{U} / 1$ 6: $333 \mathrm{U} / 1$
$3: 12,3 \mathrm{U} / 1 \quad 7: 1000 \mathrm{U} / 1$
$4: 37 \mathrm{U} / 1$

Verwendete Kontrollen

1) Anti- $\mathrm{HB}_{\mathrm{s}}$-Standard der Behringwerke Marburg, $30 \mathrm{U} / \mathrm{l}$, für den Enzygnost ${ }^{\circledR}$-Anti-HB

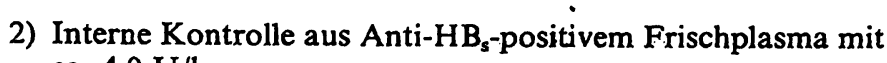
ca. $4.0 \mathrm{U} / 1$ 
3) Zwei Anti-HB,-negative Kontrollen aus verschiedenen Pools

4) Interne Kontrolle mit $750 \mathrm{U} / 1$

Auf jeder ersten, dritten etc. Platte wurden Standard und Kontrollen, auf jeder zweiten, vierten etc. Platte drei Kontrollen mitgeführt.

\section{Durchführung des Assays}

\section{J. Schritt}

Pipetticren der Standards, Kontrollen und Proben $(200 \mu \mathrm{l})$; Zugabe von je 1 Kugel pro Vertiefung;

Abdecken mit Folic; Inkubation auf Rotationsinkubator (170 $\min ^{-1}$ ) für $1,5 \mathrm{~h}$ bei Raumtemperatur;

Waschen der Kugeln mit $2 \times 6 \mathrm{ml} \mathrm{H}_{2} \mathrm{O}$

\section{Schritt}

Zugabe von $200 \mu$ des $\mathrm{HB}_{\text {- }}$-Ag-Tracers,

$1: 3000$ verdünnt in phosphatgepuffertem $\mathrm{NaCl}$-Tween $20+60$ g/l Human-Scrumalbumin;

Abdecken mit Folic;

Inkubation für $1 \mathrm{~h}$ auf dem Rotationsinkubator bei Raumtemperatur;

Waschen wie im ersten Schritt; Überführen der Kugeln in Meßröhrchen;

Zugabe von $300 \mu l$ cincr $5 \mathrm{ml} / 1$ Katalase-Lösung (stabilisiert mit $0,15 \mathrm{~mol} / 1 \mathrm{NaN}_{3}$ ) pro Röhrchen;

Messung im Luminometer mit 12 s Integrationszeit;

anschließend computergestützte Erstellung einer Standardkurve und quantitative Bestimmung der unbekannten Proben an der Standardkurve.

\section{Ergebnisse}

\section{Spezifität des Verfahrens}

Vergleichsmessungen von 300 Seren mit dem Behring Anti-HBs-Enzygnost ${ }^{\text {TO }}$ (qualitativ) ergaben bis auf vier Seren übereinstimmende Ergebnisse: Diese vier Seren wurden im Enzygnost ${ }^{\$}$ zunächst als negativ bestimmt, reagierten im ILMA mit $2-4 \mathrm{U} / 1$ positiv. In mehrfachen Nachmessungen maß der Enzygnost ${ }^{\circ}$ diese Seren teils negativ, teils positiv. Daher sind die Diskrepanzen der Meßergebnisse auf dic höhere Empfindlichkeit des ILMA gegenüber dem Enzygnost zurückzuführen.

Die Anwesenheit von anderen Hepatitis B-VirusMarkern ( $\mathrm{HBs} \mathrm{Ag}$, Anti $\mathrm{HBc}, \mathrm{HBe} \mathrm{Ag}$, Anti $\mathrm{HBe}$ ) beeinflußten die Meßergebnisse ebensowenig wie Hepatitis A-Virus-Serum-Marker.

\section{Empfindlichkeit}

Die untere Nachweisgrenze wurde durch die Messung von 20 anti- $\mathrm{HB}_{8}$ negativen Seren, Ermittlung des Mittelwertes der Messungen und anschließende Addition der dreifachen Standardabweichung zum Mittelwert ermittelt. Sie lag bei 1.3 U/l Anti-HB, (Mittelwert 23 Relative Light Units, Standardabweichung 0.98 Rc- lative Light Units: Cutoff bei 26 Relative Light Units; die Impulsrate des $1.3 \mathrm{U} / \mathrm{l}-\mathrm{Standards}$ betrug im Mittel von 20 Messungen 35 Relative Light Units).

\section{Intra-assay-Varianz}

Die Präzision in der Serie wurde durch Messung der gleichen Probe zwangzigmal hintereinander crmittelt. Sie lag bei VK $=8 \%$.

\section{Inter-assay-Varianz}

Die Präzision von Tag zu Tag wurde durch Messung der angegebenen Proben in zwanzig Ansätzen an verschicdenen Tagen ermittelt. Sic lag im Mittcl bei $\mathrm{VK}=7 \%$ (s. Tab. 1).

Dic durchschnittliche Doppelwertpräzision (Compound Precision Profile) ist der Tabelle $2 z u$ entnehmen.

Die Meßsignalstabilität der Standardkurvenpunkte zeigt die Tabelle 3.

Tab. 1. Intcrassayvarianz des Anti-HB $B_{b}-$ ILMA

\begin{tabular}{lcccl}
\hline Serum & $\begin{array}{l}\text { Sollwert } \\
(U / 1)\end{array}$ & $\begin{array}{l}\text { MeßBwert } \\
(U / 1)\end{array}$ & s (RLU)* & VK (\%) \\
\hline I & 4 & 4 & 0,32 & 8,11 \\
II & 40 & 38.9 & 2,69 & 6,92 \\
III & 750 & 762 & 40,0 & 5,26 \\
\hline
\end{tabular}

* Relative Light Units

Tab. 2. Durchschnittliche Doppelwertpräzision des Anti-HBaILMA

\begin{tabular}{rcl}
\hline Meßbereich (U/I) & $\begin{array}{l}\text { Anzahl der } \\
\text { Wertepaarc }\end{array}$ & $\begin{array}{l}\text { Mittlerer VK } \\
(\%)\end{array}$ \\
\hline $1,3-10$ & 74 & 2,73 \\
$11-100$ & 112 & 2,82 \\
$101-500$ & 82 & 2,95 \\
$501-1000$ & 46 & 2,95 \\
$1,3-1000$ & 314 & 2,85 \\
\hline
\end{tabular}

Tab. 3. Meßsignalstabilität der Standardkurvenwerte des AntjHB,-ILMA, crmittelt über 20 Ansätzc

\begin{tabular}{cccc}
\hline $\begin{array}{l}\text { Standard } \\
(\mathrm{U} / \mathrm{l})\end{array}$ & $\begin{array}{l}\text { Mittleres } \\
\text { Signal } \\
(\mathrm{RLU})\end{array}$ & $\begin{array}{l}\text { Standard- } \\
\text { abweichung } \\
\text { (RLU)* }\end{array}$ & VK (\%) \\
\hline 1,3 & 35 & 2,25 & 6,40 \\
4,1 & 58 & 2,64 & 4,57 \\
12,3 & 127 & 7,84 & 6,20 \\
37 & 311 & 13,7 & 4,41 \\
111 & 869 & 19,6 & 2,26 \\
333 & 2176 & 93,0 & 4,27 \\
1000 & 4190 & 174 & 4,14 \\
\hline
\end{tabular}

* Relative Light Units 
Das Verhältnis höchster Standard zu Anti-HBs negativen Seren in Relative Light Units (Dynamik) beträgt ca. $180: 1$.

\section{Richtigkeit des Verfahrens}

Die Richtigkeit der Meßergebnisse wurde durch eine Vergleichsmessung mit dem ABAU-Q ${ }^{\circledR}$ (Sorin Biomedica, S. P. A. I-13040 Saluggia) ELISA überprüft, der Anti-HB $\mathrm{s}_{\mathrm{s}}-$ Konzentrationen von 5-160 U/1 mißt. Innerhalb dieses Meßbereichs lag der Korrelationskoeffizient bei 0,979 ILMA $=1,97+1,28 \times$ EIA; $\mathrm{EIA}=8,33+0,602 \times$ ILMA) für $\mathrm{n}=37$.

\section{Diskussion}

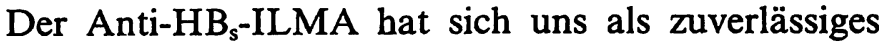
und stabiles Meßverfahren erwiesen.

Die Abweichungen von den qualitativen Meßergebnissen des Anti-HB ${ }_{\mathrm{s}}$-Enzygnost ${ }^{\circledR}$ sind offenbar (s. o.) auf die höhere Empfindlichkeit des ILMA und nicht auf unspezifische Reaktionen zurückzuführen.

Ein bis jetzt noch nicht gelöstes Problem stellt der bei sehr hohen Anti-HBs-Titern auftretende High-DoseHook-Effekt dar. Einige Seren mit Konzentrationen von 1500 bis $100000 \mathrm{U} / 1$ Anti-HB s $_{\text {s }}$ ergaben unverdünnt Meßwerte bis herab zu $350 \mathrm{U} / 1$, so daß es sich empfiehlt, unbekannte Proben in zwei Verdünnungsstufen zu messen (z. B. 1:1 und 1:100). Korrelieren die Meßwerte dieser Verdünnungen nicht, so sollten noch höhere Verdünnungsstufen gemessen werden.

Die Vorteile der vorgestellten Methode gegenüber anderen, nicht isotopischen Verfahren zur Erfassung von Anti-HB $\mathrm{B}_{\mathrm{s}}$ liegen vor allem in zwei Punkten:

1) Der ILMA ist in insgesamt ca. zwei bis drei (je nach Probenanzahl) Stunden durchführbar. Der En- zygnost ${ }^{\oplus}$ und der ABAU-Q ${ }^{\oplus}$ ELISA benötigen dagegen 24-26 Stunden zur Durchführung und sind daher nicht an einem Arbeitstag durchzuführen.

2) Der Meßbereich des ILMA beträgt 1,3-1000 U/1 gegenüber Meßbereichen von 2-30 U/1 (Enzygnost ${ }^{\circledR}$ ) bzw. 5-160 U/1 (ABAU-Q ${ }^{\circledR}$ ). Diese Differenz ist deshalb praktisch so interessant, weil die Anti-HB ${ }_{\mathbf{s}}$ Konzentrationen der Proben von nicht nachweisbar bis $\mathrm{zu}$ mehreren Hunderttausend $\mathrm{U} / 1$ reichen. Um diese große Spanne zu erfassen, sind je mehr Verdünnungsstufen erforderlich, desto geringer der Meßbereich ist. Mit dem Anti-HB $\mathrm{HB}_{\mathrm{s}}$-ILMA sind Konzentrationen von $1,3-100000 \mathrm{U} / 1$ in zwei Verdünnungsstufen $(1: 1,1: 1000)$ erfaßbar. Dies ist auch in Bezug auf die Kosten der Bestimmung interessant.

Die Herstellungskosten je 100 Einzelmessungen betragen ca. $70 \mathrm{DM}$. In Anbetracht der Kit-Preise für kommerzielle Meßbestecke stellt der ILMA auch diesbezüglich eine interessante Alternative dar.

\section{Danksagungen}

Den folgenden Personen bzw. Institutionen danken wir sehr für die Überlassung von $\mathrm{HB}_{\mathrm{s}}$-Antigen: Herrn Prof. Dr. W. Gerlich, Hygiene-Institut der Universität, Kreuzbergring 57, 3400 Göttingen; der Behringwerke AG Marburg sowie Smith-Kline RIT, Belgien.

Herzlich bedanken möchten wir uns auch bei den Mitarbeitern des Instituts für Immunologie und Transfusionsmedizin der Medizinischen Universität zu Lübeck, sowie des Instituts für Med. Mikrobiologie der Medzinischen Universität zu Lübeck, die uns Seren, Kontrollen, Frischplasmakonserven etc. überlieBen und ohne die die Gewinnung der Standards mittels Plasmapherese nicht möglich gewesen wäre. Nicht unerwähnt lassen möchten wir die Mitarbeiter des Instituts für Biochemie der Medizinischen Universität zu Lübeck, die uns bei den Versuchen, $\mathrm{HB}_{\mathrm{s}}-\mathrm{Ag}$ selbst zu gewinnen, mit Rat und Rat zur Seite standen.

Diese Arbeit wurde mit Unterstützung der Deutschen Forschungsgemeinschaft (DFG) Projekt Nr. Wo 351/1-1 durchgeführt.

\section{Literatur}

1. Ginsberg, A., Conrad, M., Bancroft, W. et al. (1973) J. Lab. Clin. Med. 82, 317.

2. Lander, J., Alter, H. \& Purcell, R. (1971) J. Immunol. 106, 1166.

3. Hollinger, F. B., Adam, E., Heiberg, D. et al. (1982) In: Viral Hepatitis 1981 International Symposium (Szmuness, W., Mayward, J. W. \& Alter, H. J., eds) The Franklin Institute Press, Philadelphia, pp. 451-466.

4. Purcell, R. H. \& Gerin, J. L. (1975) Amer. J. Med. Sci. 270, 395-399.

5. Segler, K., Strohmeyer, H., Ritter, S., Gerlich, W. H. \& Thomsen, R. (1982) Develop. Biol. Standard. 54, 179- 189 (S. Karger, Basel, 1983).

6. Lander, J., Holland, P., Alter, H., Chanock, R. \& Purcell, R. (1972) J. Am. Med. Ass. 220, 1079-1082.

7. Descoeudres, C. (1984) Schweiz. Med. Wochenschr. 114, $498-505$.

8. Jilg, W., Schmidt, M., Zachoval, R. \& Deinhard, F. (1985) Dtsch. Med. Wochenschr. 110, 205-209.

9. Wood, W. G. \& Gadow, A. (1983) J. Clin. Chem. Clin. Biochem. 21, 789-797.

10. Wood, W. G., Braun, J. \& Hantke, U. (1986) Meth. Enzymol. 133, 354-365.

PD. Dr. W. G. Wood

Klinische Laboratorien

Klinik für Innere Medizin

Medizinische Uñiversität zu Lübeck

Ratzeburger Allee 160 :i

D-2400 Lübeck 1

J. Clin. Chem. Clin. Biochem. / Vol. 26, 1988 / No. 9 